\title{
Improving the Discrimination of Hand Motor Imagery via Virtual Reality Based Visual Guidance
}

\author{
Shuang Liang a ${ }^{\mathrm{a}}$, Kup-Sze Choi ${ }^{\mathrm{b}}$, Jing Qin ${ }^{\mathrm{c}}$, Wai-Man Pang ${ }^{\mathrm{d}}$, Qiong Wang ${ }^{\mathrm{a}}$, Pheng-Ann Heng ${ }^{\mathrm{e}}$ \\ ${ }^{a}$ Shenzhen Institutes of Advanced Technology, Chinese Academy of Sciences \\ ${ }^{b}$ School of Nursing, The Hong Kong Polytechnic University \\ ${ }^{c}$ School of Medicine, Shenzhen University \\ ${ }^{d}$ The Department of Computer Science, Caritas Institute of Higher Education, Hong Kong \\ ${ }^{e}$ The Department of Computer Science and Engineering, The Chinese University of Hong Kong
}

\begin{abstract}
While research on the brain-computer interface (BCI) has been active in recent years, how to get high-quality electrical brain signals to accurately recognize human intentions for reliable communication and interaction is still a challenging task. The evidence has shown that visually guided motor imagery (MI) can modulate sensorimotor electroencephalographic (EEG) rhythms in humans, but how to design and implement efficient visual guidance during MI in order to produce better event-related desynchronization (ERD) patterns is still unclear. The aim of this paper is to investigate the effect of using object-oriented movements in a virtual environment as visual guidance on the modulation of sensorimotor EEG rhythms generated by hand MI. To improve the classification accuracy on MI, we further propose an algorithm to automatically extract subject-specific optimal frequency and time bands for the discrimination of ERD patterns produced by left and right hand MI. The experimental results show that the average classification accuracy of object-directed scenarios is much better than that of non-object-directed scenarios $(76.87 \%$ vs. $69.66 \%)$. The result of the t-test for measuring the difference between them is statistically significant ( $p=0.0207)$. When compared to algorithms based on fixed frequency and time bands, contralateral dominant ERD patterns can be enhanced by using the subject-specific optimal frequency and time bands obtained by our proposed algorithm. These findings have the potential to improve the efficacy and robustness of MI-based BCI applications.
\end{abstract}

Keywords:

Brain-computer interface, Hand motor imagery, Visual Guidance, Subject-specific frequency and time bands, Event-related desynchronization, Virtual Reality

\section{Introduction}

Brain computer interface (BCI) is a communication pathway between human intentions and the external world via the translation of electrical brain signals into messages or commands without passing through peripheral nerves or muscle tissues (Wolpaw et al., 2002; Daly \& Wolpaw, 2008). Because of its great potential in areas such as neuroprosthetics, rehabilitation, and entertainment, the scientific community has been actively studying BCI.

However, most BCI applications to date, especially the ones involving complex control tasks, cannot be widely applied in practice due to low reliability in the translation of spontaneous scalp-recorded electroencephalogram (EEG) signals to user messages and commands, which results in incorrect recognition of actual human intentions. The performance of intention recognition is partly due to the accuracy of the EEG signal processing algorithms. To this end, much research has been

Email addresses: shuang. liang@siat .ac.cn (Shuang Liang), thomasks.choi@polyu.edu.hk (Kup-Sze Choi), jing.qin@szu.edu.cn (Jing Qin), wmpang@gmail.com (Wai-Man Pang), wangqiong@siat.ac.cn (Qiong Wang), pheng@cse. cuhk.edu.hk (Pheng-Ann Heng) dedicated to the development of signal processing techniques to improve EEG feature extraction and classification for better recognition performance. For example, Zhang et al. proposed a multiway extension of canonical correlation analysis (MCCA) (Zhang et al., 2013a) and a multiset CCA (Zhang et al., 2014a) method to improve steady-state visual evoked potential (SSVEP) recognition. Higashi et al. proposed a discriminative filter bank common spatial pattern (DFBCSP) to effectively extract discriminative features for motor imagery (MI) based BCI (Higashi \& Tanaka, 2013). Another extension of CSP based on Kullback-Leibler divergence (KLCSP) was also introduced for improving MI classifications (Arvaneh et al., 2013). More recently, Zhang et al. further proposed a sparse filter band CSP (SFBCSP) for optimizing spatial patterns to obtain better MI classification accuracy (Zhang et al., 2015).

More critically, the quality of the EEG signals produced by users with a BCI system is a key factor affecting the accuracy of the recognition performance. Effective training approaches are needed to ensure that users perform the desired mental tasks, or even the most advanced signal processing algorithms will not be able to correctly identify human intentions. It is therefore important to investigate approaches and protocols for training 
users to produce quality EEG signals and control BCI systems. However, relatively little attention was paid to this area in early work.

In recent years, it has been demonstrated that motor imagery (MI) - mental rehearsals without overt movements - offer promise for motor function recovery (Jackson et al., 2001; Prasad et al., 2010), where BCI systems can be developed by capitalizing upon MI-based mental activities. The underlying reason for using $\mathrm{MI}$ in BCIs is that by imaging a unilateral movement, it is able to elicit contralateral dominant eventrelated desynchronization (ERD), as well as simultaneous ipsilateral event-related synchronization (ERS) under certain circumstances (Pfurtscheller \& Lopes da Silva, 1999; Leocani et al., 1997; Pfurtscheller \& Neuper, 1997). For example, hand MI can induce activities in $m u(8-13 \mathrm{~Hz})$ and beta $(8-25 \mathrm{~Hz})$ rhythm (McFarland et al., 2000), where the detectable sensorimotor rhythm (SMR) amplitude attenuation and enhancement are examples of ERD and ERS, respectively. Dominant ERD appears over the contralateral cortical area during the preparation and imagination of movement, while ERS appears over the ipsilateral hemisphere after the termination of physical movement (Pfurtscheller et al., 1997). Therefore, during cortical activation/deactivation, ERD or ERS can be employed as features to classify various MI tasks (Pfurtscheller et al., 1998, 2006). In this paper, we focus on the ERD of hand MI.

Research has been conducted on SMR oscillation phenomena during the observation of hand movements (Oberman et al., 2007; Ertelt et al., 2007; Babiloni et al., 2002). In an animal study reported by Rizzolatti et al. (1996), it was found that a mirror neuron system exists in monkey brains during the observation of hand movements by other monkeys. Further evidence was also obtained to show that such a mirror neuron system also exists in humans (Pfurtscheller et al., 1999), proving that visually guided MI can modulate sensorimotor EEG rhythms in humans. Similar findings were obtained from the observation of leg movements (Peter et al., 2010), where visual guidance was found to improve the discrimination of ERD patterns during MI tasks.

Further investigations have also been conducted to study the effect of the contexts and presentations of visual guidance on MI. For example, it was found in a study that during MI, stronger ERD patterns were induced from watching a moving hand than from watching a moving cube (Pfurtscheller et al., 2007), which enhanced the accuracy in EEG signal recognition. In a study investigating the effect of abstract and realistic avatars, users were guided to perform object-directed hand MI tasks with a progress bar and a hand as visual cues, respectively. The results showed that no significant difference in BCI performance (Neuper et al., 2009). Muthukumaraswamy et al. (2004) reported that the $m u$ rhythm during MI was more suppressed during the observation of object-directed grips when than of empty grips, supporting the view that the mirror neuron system can specifically respond to the observation of object-oriented actions. However, discrimination of left and right hand MI tasks could not be achieved with the ERD patterns obtained in the study. In summary, how to design an efficient visual guidance system during hand MI in order to produce better ERD patterns to enhance the user training performance is still unclear.

On the other hand, considering the variations in EEG patterns resulting from differences in the MI ability of individual subjects or differences in MI strategies, it is necessary to investigate the ERD or ERS patterns of individual subjects in order to further improve the classification accuracy on MI. In addition, there is evidence that the time and frequency characteristics of cortical rhythms components can vary widely (Neuper \& Pfurtscheller, 2001). Therefore, it is desirable to identify ERD or ERS patterns from both optimal time and frequency bands to achieve more robust recognition. In this paper, we will investigate the extraction of subject-specific patterns in both the time and frequency domains in order to improve the discrimination of different MI tasks.

To solve the above-mentioned problems, this paper aims to investigate the effect of visual guidance in the form of objectdirected movements on the discrimination of left and right hand MI. The results will provide insight into the design and development of efficient BCI and training strategies. In addition, we propose an algorithm to automatically extract subject-specific optimal frequency and time bands for the discrimination of ERD patterns. The results of experiments demonstrate that observing virtual object-directed movements during MI can induce ERD patterns that can be more accurately discriminated for classifying left and right hand MI. The average classification accuracy for all subjects increased by about $7 \%$ when virtual object-directed movements were observed than when nonobject-directed movement were observed. The result of the ttest also indicated that the difference in classification performance between observing object-guided movements and nonobject-directed movement was significant ( $p=0.0207)$. In addition, the contralateral dominant ERD patterns were found to be enhanced by using the optimal frequency and time bands obtained with our proposed algorithm, and the optimal frequency and time bands were generally within a relatively stable range. These findings in this study can serve as a reference to guide the design and development of BCI applications.

\section{Methods}

\subsection{Subjects and Data Acquisition}

Seven subjects, consisting of 4 males and 3 females, between the ages of 25 and 30, were recruited for the experiments. During the experiments, they were asked to sit in a quiet and dim room, and to look at a computer screen from a distance of about $50 \mathrm{~cm}$. All of the subjects were right-handed, with normal vision and without neurological or psychiatric problems. Before the experiments, the subjects were given details of the entire experimental procedure. None of the subjects had prior background knowledge or experience of BCI and EEG.

A Biosemi ActiveTwo system with $32 \mathrm{Ag} / \mathrm{AgCl}$ electrodes was used for recording EEG data. With reference to the wellknown 10 - 20 system (Jasper, 1958), the measurement was made at a sampling rate of $256 \mathrm{~Hz}$. The offset at each electrode was between $\pm 25 \mathrm{mV}$. The electrodes involved were mainly around the left and right hemispheres $(\mathrm{C} 3, \mathrm{C} 4, \mathrm{Cz}, \mathrm{F} 3, \mathrm{~F} 4$, 


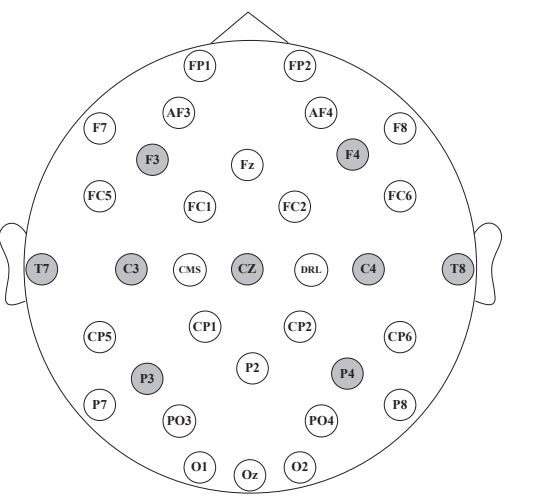

Figure 1: EEG electrode positions used in the Biosemi ActiveTwo system with $32 \mathrm{Ag} / \mathrm{AgCl}$ electrodes, and the shadowed electrodes are the ones corresponding to the sensorimotor area.

T7, T8, P3, and P4, see Figure 1), through which EEG signals corresponding to the sensorimotor area were obtained. Trigger signals in the form of rectangular pulses sent from another computer were also recorded to label different experimental trials (a trial includes a rest period and a motor imagery period, which will be introduced in the next subsection). The accuracy of the time interval divided by the trigger signals was above $96 \%$.

\subsection{Experimental Procedure}

Each subject was asked to take part in two scenarios of the experiment: (1) a display of non-object-directed hand movements in a virtual reality (VR) environment, (2) a display of object-directed hand movements in a VR environment. Snapshots of these two scenarios are illustrated in Figure 2(a) and Figure 2(b), respectively. During the experiment, the subject$\mathrm{s}$ were required to imagine left or right hand movements, with the two scenarios displayed on the computer screen from a firstperson perspective, while remaining relaxed and avoiding any body motions.

The paradigm of a single-trial MI in the experimental process is illustrated in Figure 3. For a single-trial MI, it consists of $a$ rest period of 2 seconds and a motor imagery period of 4.5 seconds. In particular, the motor imagery period is divided into the three movements, each of which corresponds to a complete hand opening/grasping MI. The trajectory of hand movements was not changed between each trial.

In the whole experimental procedure, an auditory cue was first played to signify the start of the experimental process. In the first 5 seconds, i.e., the preparation stage, a black screen was displayed. Then, the subject was presented with a specific hand movement task shown on the screen, and started to imagine the task for 4.5 seconds, until $9.5 \mathrm{~s}$ has elapsed. This was referred to as the motor imagery period. This was followed by a rest period of 2 seconds, when a black screen appeared again the subject could take a short break during this period. Here, a trial was defined as one rest period plus one motor imagery period. The motor imagery and rest epochs were repeated 40 times, including 20 trials for left MI and 20 trials for right MI. That is, there were a total of 40 trials for each scenario (nonobject-directed or object-directed hand movements).

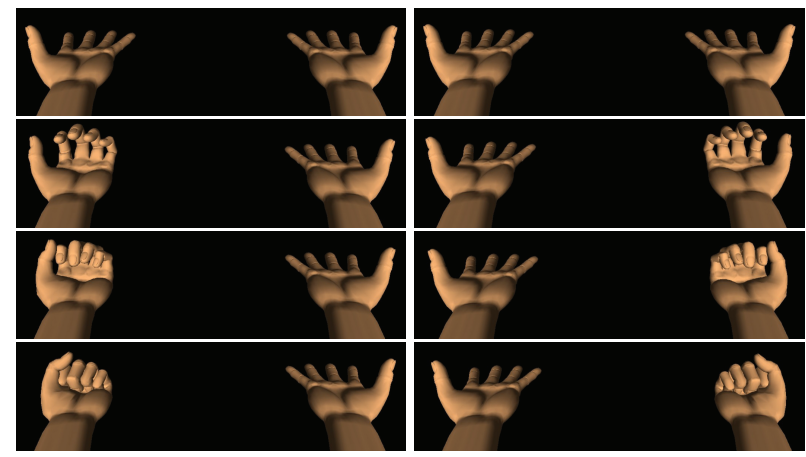

(a)
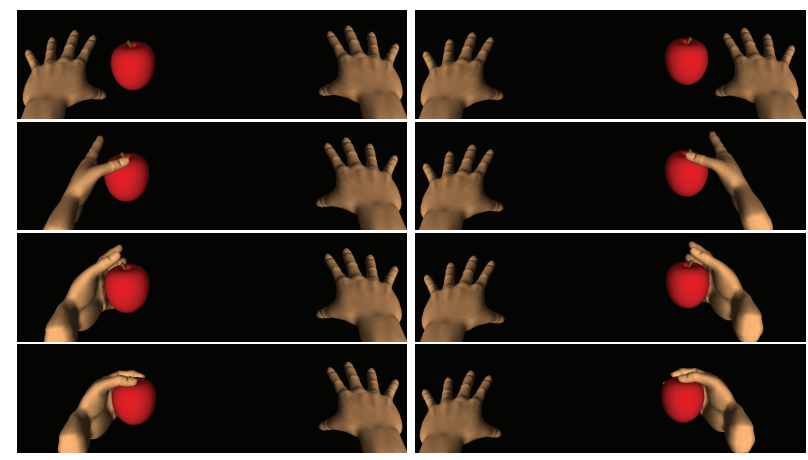

(b)

Figure 2: Snapshots of two scenarios. The subjects were asked to imagine left or right hand movements with (a) non-object-directed scenario and (b) objectdirected scenario.

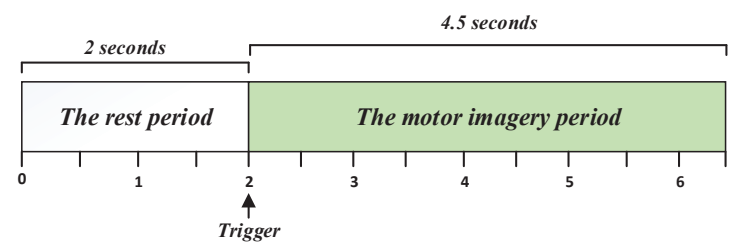

Figure 3: The paradigm of a single-trial MI in the experimental process.

An auditory cue was played at the end of the experimental process. The subjects were randomly asked to go through the experimental process with and without the presence of objectdirected hand movements as visual guidance for hand MI. In the end, the experiential process was repeated for 5 runs for each scenario. In between each run, the subjects were given 2 minutes of rest to relieve experimental fatigue.

All together, each subject was required to carry out 400 trials, with 200 trials for non-object-directed MI and 200 trials for object-directed hand MI. Eventually, raw experimental data for 2800 trials were obtained from the seven subjects.

\subsection{Data Analysis}

To analyze the experimental data, the raw EEG signals were first filtered using the surface Laplacian filter and the bandpass filter. Subsequently, we applied Hilbert-Huang Transform to 


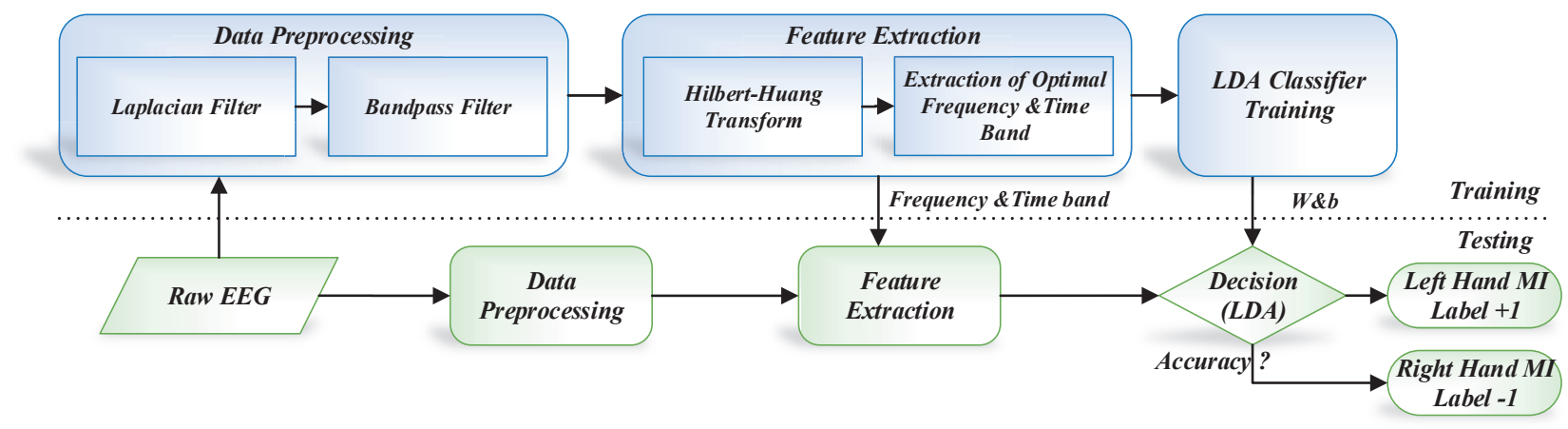

Figure 4: Illustration of the signal processing and analysis procedure.

extract signal features in both the time and frequency domains. The subject-specific optimal frequency and time bands were further extracted using the proposed algorithm. Linear discriminant analysis (LDA) was then used for classifier training. Finally, data analysis was performed based on statistical methods. The pipeline of our signal analysis procedure is shown in Figure 4 .

\subsubsection{Data Preprocessing}

As decreases in band power of the mu rhythm during MI tasks are centered on the area of the sensorimotor cortex (Pfurtscheller et al., 2006), the channels surrounding C3 and C4 were taken into account (see in Figure 1). In this regard, we first removed the data obtained from other channels and then processed the signals at the channels of interest around $\mathrm{C} 3(\mathrm{~F} 3$, $\mathrm{T} 7, \mathrm{P} 3, \mathrm{Cz})$ and $\mathrm{C} 4(\mathrm{~F} 4, \mathrm{~T} 8, \mathrm{P} 4, \mathrm{Cz})$, respectively, using the surface Laplacian algorithms (McFarland et al., 1997), where we made an approximate calculation of the scalp potential at $\mathrm{C} 3$ and $\mathrm{C} 4$ by subtracting the average potential of its four neighboring channels. The EEG signals were then filtered using an 8-14 $\mathrm{Hz}$ bandpass filter.

\subsubsection{Hilbert-Huang Transform Analysis}

To extract features for further analysis in both the time and frequency domains, the Hilbert-Huang Transform (HHT) was used to identify the features in the preprocessed EEG data without uncertainty principle limitations on both domain resolutions. HHT has been shown to be viable for analyzing nonlinear and non-stationary data, especially for time-frequency-energy representations (Huang et al., 1998).

HHT is composed of two parts: empirical mode decomposition (EMD) and Hilbert spectral analysis (HSA). In our application, the EEG signals were first decomposed into a series of intrinsic mode functions (IMFs) through a sifting process.

For a preprocessed signal $X(t)$, we first identify all of the local extrema of $X(t)$, including both maxima and minima. Then, we separately connect all maxima and minima with spline lines to generate upper $u_{1}(t)$ and lower $l_{1}(t)$ envelopes. The mean of the two envelopes can be calculated as $m_{1}(t)=\left[u_{1}(t)+l_{1}(t)\right] / 2$.
The sifting process starts with the computation of the difference between the signal $X(t)$ and the $m_{1}(t)$ :

$$
\begin{aligned}
& h_{1}(t)=X(t)-m_{1}(t) . \\
& h_{11}(t)=h_{1}(t)-m_{11}(t),
\end{aligned}
$$

where $h_{1}(t)$ is treated as the data, and $h_{11}(t)$ is computed from the sifting process performed on $h_{1}(t) . m_{11}(t)$ is the mean of the upper and lower envelopes of $h_{1}(t)$. The process is repeated $k$ times until a stoppage criterion is satisfied:

$$
\begin{aligned}
& h_{1 k}(t)=h_{1(k-1)}(t)-m_{1 k}(t), \\
& c_{1}(t)=h_{1 k}(t),
\end{aligned}
$$

where $h_{1 k}(t)$ is computed from the sifting process performed on $h_{1(k-1)}(t)$. Thus, we set $c_{1}(t)$ as the first IMF that can be separated from the signal $X(t)$. The residue $r_{1}(t)$ can be obtained as:

$$
r_{1}(t)=X(t)-c_{1}(t)
$$

Next, we perform the same sifting process on the residue $r_{1}(t)$ and obtain the second IMF. Finally, the sifting process will be stopped by predetermined criteria (Huang et al., 1998). Thus, we can obtain a series of IMFs. The signal $X(t)$ can be linearly reconstructed as a collection of $n \operatorname{IMFs}\left(i m f_{i}(t)=c_{i}(t)\right)$ plus a residue $r_{n}(t)$ :

$$
X(t)=\sum_{i=1}^{n} i m f_{i}(t)+r_{n}(t) .
$$

We use the subscript $c$ as the identifier of the EEG channel positions (e.g., C3 or C4). The time series for different channels $X_{c}(t)$ can be expressed as:

$$
X_{c}(t)=\sum_{i=1}^{n} i m f_{c i}(t)+r_{c n}(t),
$$

where $\operatorname{im} f_{c i}(t)$ is the intrinsic mode function component of the signal. On the other hand, the Hilbert spectral analysis is introduced to transform each IMF component $i m f_{c i}(t)$, and the 
Hilbert spectrum of the original signal can be calculated as follows:

$$
H_{c}\left(\omega_{c}, t\right)=\operatorname{Re} \sum_{i=1}^{n} a_{c i}(t) e^{j \int \omega_{c i}(t) d t} .
$$

where $a_{c i}(t)$ is the instantaneous amplitude of $i m f_{c i}(t), \omega_{c i}(t)$ is the instantaneous frequency, and $R e$. is defined as the real part of a complex quantity.

In order to obtain data on variations in energy, the Hilbert energy spectrum and instantaneous energy are calculated by integrating the Hilbert amplitude spectrum into the frequency or time domains, respectively as follows:

$$
\begin{aligned}
& E S_{c}(\omega)=\int_{t_{1}}^{t_{2}} H_{c}^{2}(\omega, t) d t \\
& I E_{c}(t)=\int_{\omega_{1}}^{\omega_{2}} H_{c}^{2}(\omega, t) d \omega .
\end{aligned}
$$

The Hilbert energy spectrum $E S_{c}(\omega)$ is a measurement of the energy contribution from each frequency value. The instantaneous energy $I E_{c}(t)$ describes the change in signal energy over time. Given that the $I E_{c}(t)$ over the MI period is $A(t)$, and the average of the $I E_{c}(t)$ in $1 \mathrm{sec}$ reference period before MI is $R$, according to the quantification of ERD (Pfurtscheller \& Lopes da Silva, 1999), the percentage of decrease in instantaneous energy $I E D_{c}(t)$ can be calculated as:

$$
I E D_{c}(t)=\frac{A(t)-R}{R} \times 100 \% .
$$

\subsubsection{Extraction of the Optimal Time and Frequency Bands}

Because inter-subject variability in time and frequency patterns exists, it is necessary to further investigate the optimal ERD features for each subject. In this regard, we develop an algorithm to automatically extract the optimal ERD features that can be use to discriminate left and right hand MI, in both the frequency and time domains for each subject. We will detail the algorithm in the time domain while the same procedure applies to the frequency domain.

We first calculate the mean value of instantaneous energy decrease $\overline{I E D}_{c}^{\text {label }}$ over different trials of left hand MI and right hand MI, respectively. Thus, $\overline{I E D}_{c 3}^{+1}, \overline{I E D}_{c 3}^{-1}, \overline{I E D}_{c 4}^{+1}$ and $\overline{I E D}_{c 4}^{-1}$ can be obtained (hereafter, we use -1 to denote the signals of the left hand MI and +1 to denote the signals of the right hand MI; the electrode position $c$ refers to either $C 3$ or $C 4$.). We use $X_{c}^{\text {label }}$ to denote these instantaneous energies, where label $=\{-1,+1\}$ and $c=\{C 3, C 4\}$.

In order to measure the contribution of $X_{c}$ to the discrimination of left and right hand MI, we calculate the value of the Fisher separability criterion at each electrode position:

$$
F_{\text {score }}\left(X_{c}\right)=\frac{\left(E X_{c}^{+1}-E X_{c}^{-1}\right)^{2}}{\sigma_{X_{c}^{+1}}^{2}+\sigma_{X_{c}^{-1}}^{2}},
$$

where $E X_{c}$ and $\sigma_{X_{c}}^{2}$ represent the mean and variance of $X_{c}$, respectively. The optimal time band can then be sifted according
Algorithm 1 Algorithm for extracting the optimal ERD features to discriminate left and right $\mathrm{MI}$ in the time domain.

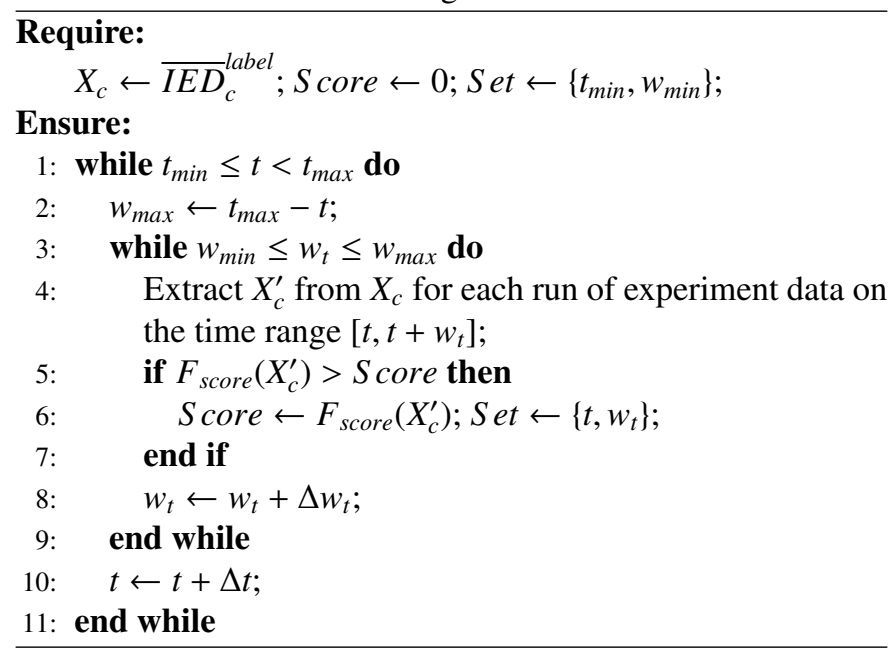

to the value of the Fisher separability criterion. We firstly initialize $\omega$ ranging from $8-14 \mathrm{~Hz}$ frequency and $t$ from 2-6 sec in a trial. Then, with the implementation of the sliding window, we can make an exhaustive search of the optimized frequency and time band for the best discrimination of the left and right hand MI.

The algorithm starts from the beginning point of the time band $t$, and the initial width of a time sliding window $w_{t}$, and iteratively performs the following two steps. In the first step, the beginning point of the time band is kept fixed and the width of the sliding window is gradually increased. The process starts from $t_{\min }$ with the width of the sliding window $w_{\min }$. The sifted value $X_{c}^{\prime}$ from the window range $\left[t_{\text {min }}, t_{\text {min }}+w_{\text {min }}\right]$ on $X_{c}$ is used to calculate the the Fisher separability criterion $F_{\text {score }}\left(X_{c}^{\prime}\right)$. The width of the sliding window is then increased by a step, and a new $F_{\text {score }}$ value of the sifted $X_{c}^{\prime}$ from the current window range on $X_{c}$ is obtained. The larger value of $F_{\text {score }}$ is saved. This process is repeated until the width of the sliding window exceeds the maximum width $w_{\max }$.

The second step of the algorithm is indeed the same as that of the first step except that the beginning point of time band is shifted to $t+\Delta t$. The optimal time band $\left[t, t+w_{t}\right]$ on $X_{c}$ can then be selected according to the maximum value of $F_{\text {score }}\left(X_{c}^{\prime}\right)$. That is, the ERD feature in this time band on $X_{c}$ is most optimal for discriminating left and right hand MI. The pseudo code of our algorithm is given in Algorithm 1.

In Algorithm 1, Score keeps the current maximum value of $F_{\text {score }} ; t$ is the beginning point of the current time range; and $w_{t}$ is the current width of the sliding window; $\Delta w_{t}$ and $\Delta t$ denote the variations in the step lengths of $w_{t}$ and $t$ in each loop, respectively.

\subsubsection{Feature Extraction}

For each trial, we use $M_{c 3}^{1}$ to represent the mean value of the percentage of the decrease in instantaneous energy $I E D_{c}(t)$ in the optimal time band at electrode position $\mathrm{C} 3$, and $M_{c 4}^{1}$ to represent the mean value at electrode position C4. $M_{c 3}^{1}$ and $M_{c 4}^{1}$ can 
Table 1: The W vectors of LDA for subjects

\begin{tabular}{rrrrrrrrr}
\hline & \multicolumn{4}{c}{ object-directed hand movements } & \multicolumn{4}{c}{ non-object-directed hand movements } \\
\hline $\mathbf{I}$ & -0.9531 & -0.2858 & -0.2494 & 0.8108 & -0.7284 & -0.0720 & -1.5831 & 0.9138 \\
$\mathbf{2}$ & 0.2802 & 0.5459 & -1.7785 & -0.5759 & -0.3790 & 1.4372 & -0.9168 & 2.4067 \\
$\mathbf{3}$ & -2.1688 & 0.8698 & -1.2624 & -0.0097 & 0.5493 & 1.2301 & -0.8720 & 1.8713 \\
$\mathbf{4}$ & 5.0117 & 2.6318 & 2.9092 & 0.5927 & 1.0190 & 1.5436 & 0.4134 & 0.2925 \\
$\mathbf{5}$ & 0.7578 & 0.3126 & -0.8928 & 0.0148 & -0.8211 & -1.0115 & 0.9121 & -1.0780 \\
$\mathbf{6}$ & -0.3952 & 1.2107 & -0.3629 & -0.4715 & -0.6440 & -0.2816 & 0.7535 & 0.4083 \\
$\mathbf{7}$ & 1.4205 & 0.6567 & 0.7260 & 0.2688 & -0.3702 & 0.3691 & 0.7549 & 0.6134 \\
\hline
\end{tabular}

be considered features in the time domain. The same process is performed in the frequency domain. We use $M_{c 3}^{2}$ to represent the mean value of the Hilbert energy spectrum $E S_{c}(\omega)$ in the optimal frequency band at the EEG electrode position $\mathrm{C} 3$, and $M_{c 4}^{2}$ to represent the value at electrode position C4. IED $(t)$ and $E S_{c}(\omega)$ can be calculated by the methods reported in the last paragraph in section 2.3.2. Thus, with the proposed algorithm, we can select the ERD features from the optimal time and frequency bands, and concatenate them into a feature vector $X=\left[M_{c 3}^{1}, M_{c 4}^{1}, M_{c 3}^{2}, M_{c 4}^{2}\right]$ for classification.

\subsubsection{Classification and Statistical Analysis}

We employ LDA for classifying the EEG signals. Given $X$ as the input of a classifier, the output is $Y=(W)^{T} X+b$, where $W$ and $b$ are respectively the weights and bias determined by the optimal ERD feature vector (Duda et al., 2001). A $5 \times 5$ cross-validation is implemented to evaluate the classification performance. For each subject, the last run including 40 trials is used for testing, and remainder 160 trials for training and cross-validation. In the cross-validation, the 128 trials are used for training and 32 trials for validation. Finally, we perform a statistical analysis to investigate the effect of virtual objectdirected movements and the optimal ERD features obtained by the proposed algorithm on the discrimination of left and right hand MI. The $W$ vectors of LDA for every subject are listed in Table 1.

\section{Results}

\subsection{ERD Feature Extraction and Analysis}

The ERD patterns of the subjects during the experimental processes were measured and analyzed. The patterns that they produced were similar. For illustration, the ERD patterns produced by a representative subject aged 25 years old during left and right hand MI, with and without using object-directed movements as visual guidance are shown in Figure 5. The patterns are presented in both the frequency and time domains.

The results of the object-directed scenario are shown in Figure 5(a)-(d). It is observed in Figure 5(a) that at C3, the Hilbert energy spectrum of the $m u$ rhythm in the frequency domain as activated by right hand MI was lower than that activated by left hand MI, while the result was reversed at C4 (Figure 5(b)). The findings are consistent with the principle of contralateral dominance (Pfurtscheller \& Lopes da Silva, 1999). The ERD patterns is also investigated by the percentage decrease in instantaneous energy relative to the 1 - second baseline power within the predefined frequency band in time domain. As shown in
Table 2: The frequency optimal band for ERD feature extraction at the C3 and $\mathrm{C} 4$ positions. For each position, the lower and upper bound of the optimal bands of seven subjects (S1-S7), as well as the average value and standard deviation of all of the subjects are listed.

\begin{tabular}{|c|c|c|c|c|c|c|c|c|}
\hline \multirow{3}{*}{$\begin{array}{c}\text { Sub. } \\
1\end{array}$} & \multicolumn{4}{|c|}{ No object-directed MI $[\mathrm{Hz}]$} & \multicolumn{4}{|c|}{ Object-directed MI $[\mathrm{Hz}]$} \\
\hline & \multicolumn{2}{|c|}{$\mathrm{C} 3$} & \multicolumn{2}{|c|}{$\mathrm{C} 4$} & \multicolumn{2}{|c|}{$\mathrm{C} 3$} & \multicolumn{2}{|c|}{$\mathrm{C} 4$} \\
\hline & 10.4 & 12.0 & 10.2 & 12.1 & 10.3 & 12.5 & 10.8 & 12.2 \\
\hline 2 & 9.6 & 11.1 & 10.9 & 12.1 & 9.9 & 11.7 & 9.9 & 12.1 \\
\hline 3 & 9.4 & 11.8 & 9.5 & 11.6 & 11.3 & 13.1 & 10.9 & 13.0 \\
\hline 4 & 9.8 & 11.8 & 10.0 & 11.6 & 11.2 & 13.5 & 12.1 & 13.8 \\
\hline 5 & 9.4 & 12.2 & 9.2 & 11.8 & 8.9 & 11.2 & 9.0 & 10.8 \\
\hline 6 & 9.3 & 11.7 & 10.0 & 12.2 & 10.6 & 12.6 & 10.4 & 12.6 \\
\hline 7 & 10.8 & 12.6 & 11.5 & 12.7 & 11.3 & 12.7 & 9.7 & 11.3 \\
\hline Avg. & 9.8 & 11.9 & 10.2 & 12.0 & 10.5 & 12.5 & 10.4 & 12.3 \\
\hline $\mathrm{SD}$ & 0.6 & 0.5 & 0.8 & 0.4 & 0.9 & 0.8 & 1.0 & 1.0 \\
\hline
\end{tabular}

Table 3: The time optimal band for ERD feature extraction at the $\mathrm{C} 3$ and $\mathrm{C} 4$ positions. For each position, the lower and upper bound of the optimal time periods of seven subjects (S1-S7), as well as the average value and standard deviation of all of the subjects are listed.

\begin{tabular}{|c|c|c|c|c|c|c|c|c|}
\hline \multirow{3}{*}{$\begin{array}{c}\text { Sub. } \\
1\end{array}$} & \multicolumn{4}{|c|}{ No object-directed MI $[s]$} & \multicolumn{4}{|c|}{ Object-directed MI $[s]$} \\
\hline & \multicolumn{2}{|c|}{$\mathrm{C} 3$} & \multicolumn{2}{|c|}{$\mathrm{C} 4$} & \multicolumn{2}{|c|}{$\mathrm{C} 3$} & \multicolumn{2}{|c|}{$\mathrm{C} 4$} \\
\hline & 2.1 & 2.8 & 3.2 & 3.8 & 2.8 & 3.5 & 2.3 & 2.9 \\
\hline 2 & 2.3 & 2.9 & 1.9 & 2.9 & 3.1 & 3.7 & 2.6 & 3.2 \\
\hline 3 & 1.9 & 2.8 & 1.9 & 2.8 & 2.2 & 3.1 & 2.0 & 2.5 \\
\hline 4 & 2.1 & 2.7 & 3.2 & 4.0 & 2.5 & 3.1 & 2.4 & 3.3 \\
\hline 5 & 1.9 & 2.7 & 2.3 & 3.3 & 2.5 & 3.4 & 2.7 & 3.3 \\
\hline 6 & 2.3 & 3.0 & 2.5 & 3.1 & 3.2 & 3.7 & 1.5 & 2.2 \\
\hline 7 & 3.0 & 3.8 & 2.1 & 2.6 & 2.8 & 3.7 & 2.1 & 2.7 \\
\hline Avg. & 2.2 & 3.0 & 2.4 & 3.2 & 2.7 & 3.5 & 2.2 & 2.9 \\
\hline SD & 0.4 & 0.4 & 0.6 & 0.5 & 0.4 & 0.3 & 0.4 & 0.4 \\
\hline
\end{tabular}

Figure 5(c) and Figure 5(d), at C3, the instantaneous energy decrease during right hand MI was more than that during left hand MI. By contrast, at C4, the instantaneous energy decrease during right hand $\mathrm{MI}$ was less than that during left hand MI. As with the analysis in the frequency domain, ERD contralateral dominance can be also revealed in the time domain.

The corresponding results of the non-object-directed scenari$\mathrm{o}$ are shown in Figure 5(e)-(h), where the decrease in energy in both the frequency and time domains is consistent with the ERD patterns in the object-directed scenario. In the time domain, although the curves of the left hand and right hand MI gradually converged at the end of the MI time period (Figure 5(g), (h)), there were distinct differences between the two scenarios in the percentage decrease in instantaneous energy decrease. However, it is observed that, in the frequency domain, the difference in ERD patterns between left and right hand MI in the objectdirected scenario was much more significant than that in the non-object-directed scenario. In fact, it is difficult to discriminate between the ERD patterns in the left and right hand MI at $\mathrm{C} 3$ in the non-object-directed scenario (Figure 5(e)). From the results, it is speculated that when virtual object-directed movement is provided as visual guidance during MI, the neural activity in the brain is more significant, making discriminating between left and right hand MI easier, especially in the frequency domain.

The inter-subject variability in ERD patterns was also investigated during the object-directed and non-object-directed scenarios. The averaged optimal frequency bands obtained for ERD feature extraction and discrimination at C3 and C4 are 


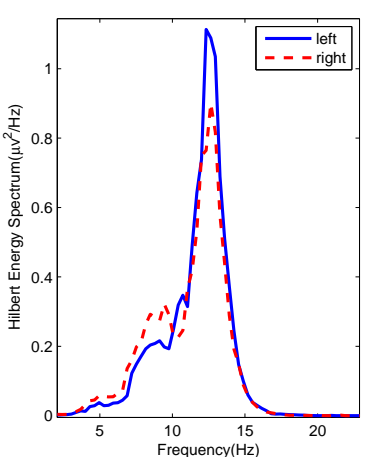

(a)

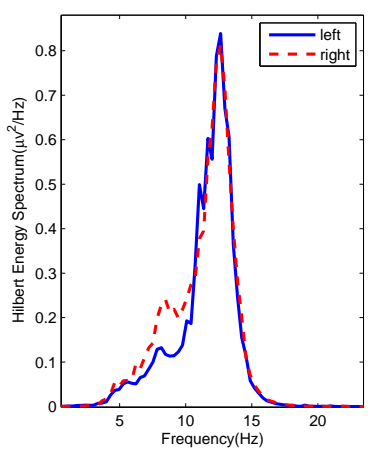

(e)

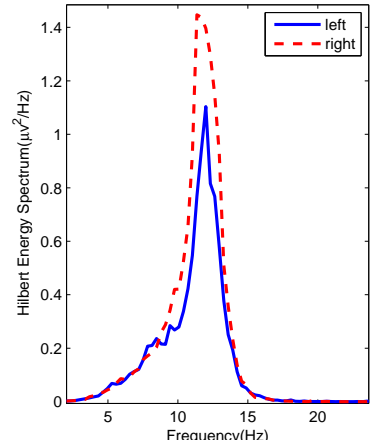

(b)

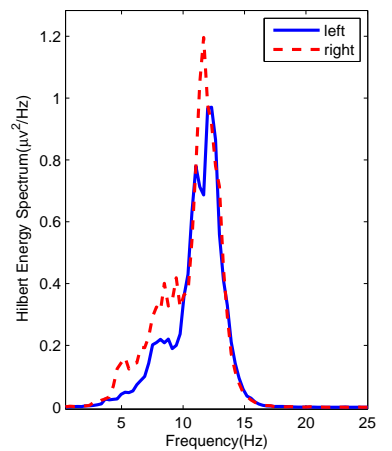

(f)

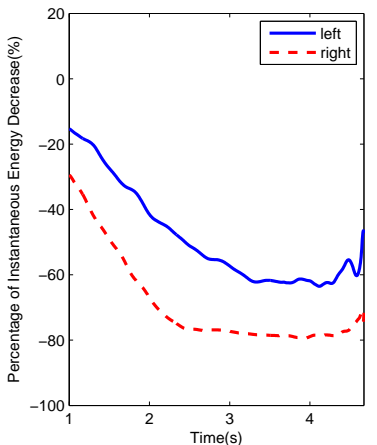

(c)

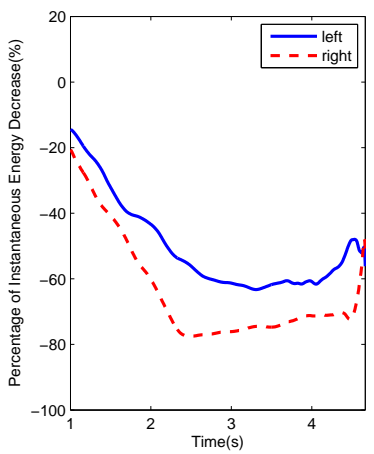

(g)

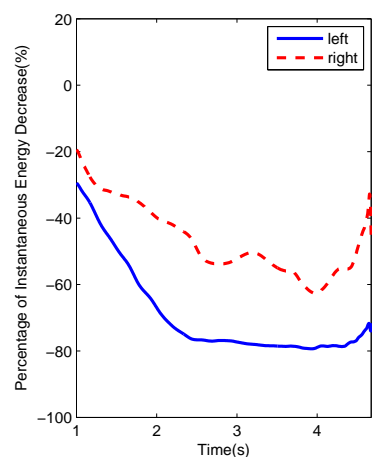

(d)

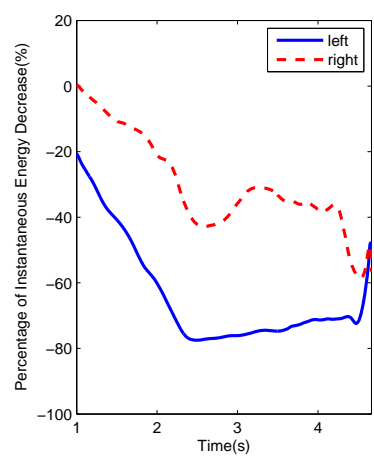

(h)

Figure 5: Contralateral dominance ERD patterns at C3 and C4 during left and right hand motor imagery in the two scenarios. The frequency and time patterns of the object-directed scenario are illustrated in (a) the frequency domain at C3, (b) the frequency domain at C4, (c) the time domain at C3, and (d) the time domain at $\mathrm{C} 4$, while those of the non-object-directed scenario are illustrated in (e)-(h).

Table 4: The cross-validation and testing accuracy (Mean \pm SD) for different subjects (S1-S7) and scenarios without optimization. The average values of all of the subjects are listed in the last line.

\begin{tabular}{|c|c|c|c|c|c|c|c|c|}
\hline \multirow{3}{*}{ Sub. } & \multicolumn{4}{|c|}{ Non-object-directed [\%] } & \multicolumn{4}{|c|}{ Object-directed [\%] } \\
\hline & \multicolumn{2}{|c|}{ Cross-validation } & \multicolumn{2}{|c|}{ Testing } & \multicolumn{2}{|c|}{ Cross-validation } & \multicolumn{2}{|c|}{ Testing } \\
\hline & Mean & SD & Mean & SD & Mean & SD & Mean & SD \\
\hline 1 & 68.54 & 6.02 & 54.88 & 10.57 & 72.17 & 5.59 & 70.80 & 9.25 \\
\hline 2 & 77.88 & 4.91 & 57.33 & 5.32 & 79.85 & 4.43 & 62.93 & 4.58 \\
\hline 3 & 79.97 & 4.45 & 68.48 & 8.24 & 82.13 & 5.26 & 81.60 & 5.55 \\
\hline 4 & 73.20 & 7.60 & 49.04 & 5.89 & 77.59 & 4.99 & 54.72 & 8.02 \\
\hline 5 & 69.17 & 5.68 & 50.72 & 7.67 & 71.90 & 6.93 & 56.16 & 9.17 \\
\hline 6 & 77.83 & 5.23 & 57.92 & 7.01 & 80.07 & 3.91 & 72.00 & 4.89 \\
\hline 7 & 69.47 & 5.76 & 54.48 & 9.48 & 69.07 & 5.99 & 60.40 & 9.55 \\
\hline Avg. & 73.72 & 5.66 & 56.12 & 7.74 & 76.11 & 5.30 & 65.52 & 7.29 \\
\hline
\end{tabular}

shown in Table 2. The lower and upper bounds of the optimal frequency bands of each subject, as well as the average value and standard deviation of all seven subjects are listed in the table. It is found that the optimal frequency band is in the range of $10 \mathrm{~Hz}$ to $12 \mathrm{~Hz}$, which is consistent with the upper $m u 2$ band (Pfurtscheller et al., 2000). The result demonstrates that the upper frequency component allows for more discriminative ERD patterns than the lower frequency component. Therefore, simply utilizing the general $m u(8-14 \mathrm{~Hz})$ frequency band to extract feature may be not a good choice for ERD feature extraction.

Table 3 shows the average optimal time periods for ERD fea-
Table 5: The cross-validation and testing accuracy (Mean \pm SD) for different subjects (S1-S7) and scenarios with optimization. The average values of all of the subjects are listed in the last line.

\begin{tabular}{|c|c|c|c|c|c|c|c|c|}
\hline \multirow{3}{*}{ Sub. } & \multicolumn{4}{|c|}{ Non-object-directed [\%] } & \multicolumn{4}{|c|}{ Object-directed [\%] } \\
\hline & \multicolumn{2}{|c|}{ Cross-validation } & \multicolumn{2}{|c|}{ Testing } & \multicolumn{2}{|c|}{ Cross-validation } & \multicolumn{2}{|c|}{ Testing } \\
\hline & Mean & SD & Mean & SD & Mean & SD & Mean & SD \\
\hline 1 & 76.19 & 4.72 & 73.20 & 8.87 & 77.84 & 5.59 & 75.12 & 6.65 \\
\hline 2 & 77.33 & 4.53 & 64.08 & 5.16 & 80.27 & 4.89 & 74.24 & 6.21 \\
\hline 3 & 79.50 & 4.83 & 70.88 & 6.81 & 88.28 & 3.83 & 86.32 & 5.63 \\
\hline 4 & 85.22 & 3.91 & 73.12 & 3.06 & 93.91 & 2.84 & 78.16 & 5.75 \\
\hline 5 & 75.82 & 6.06 & 61.62 & 8.56 & 78.50 & 4.70 & 70.20 & 8.35 \\
\hline 6 & 76.54 & 5.08 & 75.70 & 3.85 & 82.02 & 5.24 & 75.64 & 3.45 \\
\hline 7 & 75.60 & 5.67 & 69.04 & 8.85 & 80.26 & 4.91 & 78.40 & 5.41 \\
\hline Avg. & 78.03 & 4.97 & 69.66 & 6.45 & 83.01 & 4.57 & 76.87 & 5.92 \\
\hline
\end{tabular}

ture extraction and discrimination at $\mathrm{C} 3$ and $\mathrm{C} 4$. The lower and upper bounds of the optimal time periods of each subject, as well as the average and standard deviation of all seven subjects are listed in the table. It is found that the optimal time periods are generally in the range of $2.2 s$ to $3.5 s$ after the start of the MI period, which is approximately at the mid-point of the MI period. The results are reasonable since there is usually a delay in the decrease in EEG power after the MI task begins. Furthermore, the result also shows that ERD features may not exist during the whole MI period. Therefore, it is necessary to extract a specific time period for ERD feature extraction and 


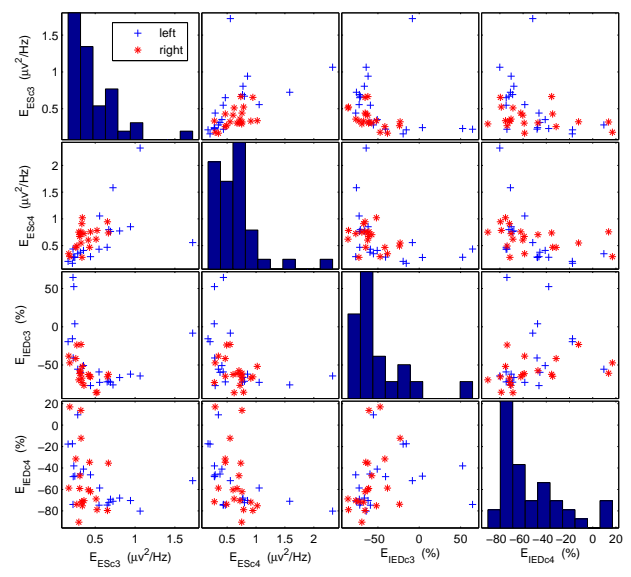

(a)

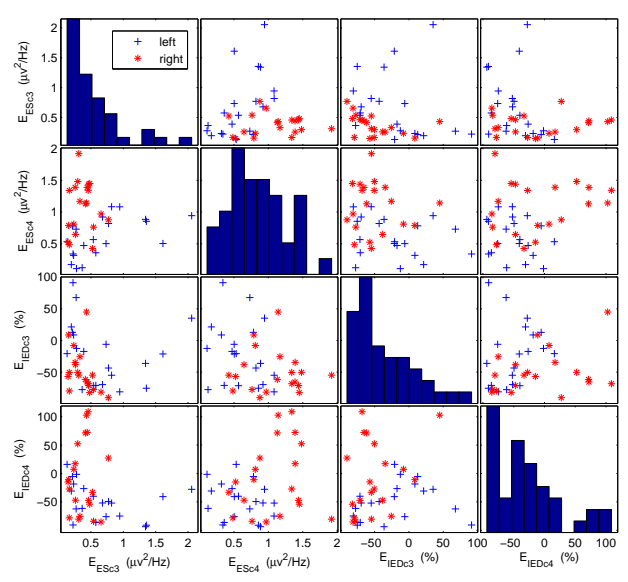

(c)

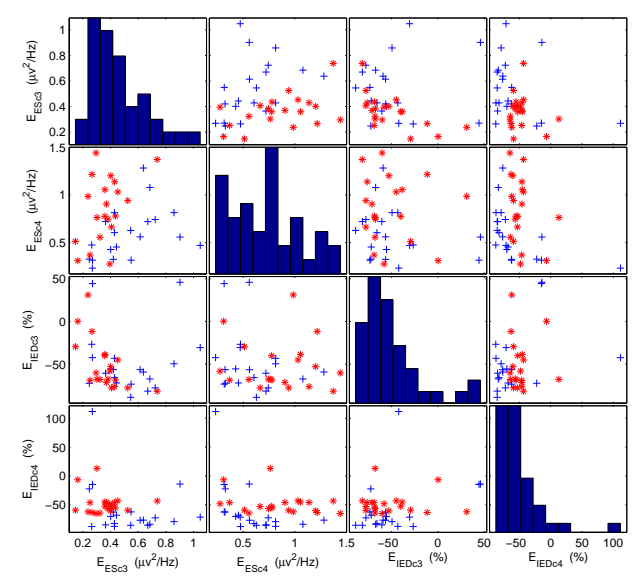

(b)

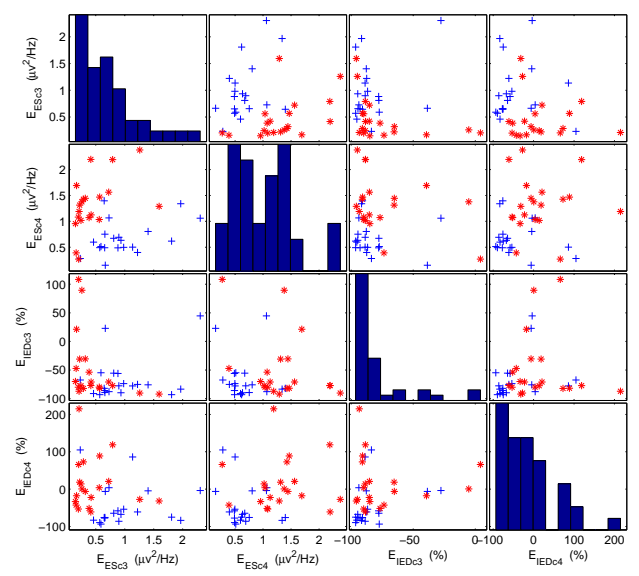

(d)

Figure 6: The scatter plot of samples of the feature vector $X$ without and with optimization in the two scenarios. The scatter plot of samples with the whole $m u$ frequency band $(8-14 \mathrm{~Hz})$ and the MI time period $(0-4.5 s)$ in (a) the non-object-directed scenario compared with those in (b) the object-directed scenario. The corresponding results of samples with optimization are shown in (c) the non-object-directed scenario and (d) the object-directed scenario.

discrimination.

We further present the scatter plot of samples of the feature vector $X$ with all possible combinations of the four components of the feature vector $X$ against each other. The histograms are placed alone the diagonals, as shown in Figure 3.1. The scatter plot of samples with the whole $m u$ frequency band $(8-14 \mathrm{~Hz})$ and the MI time period $(0-4.5 s)$ are shown in Figure 6(a)(b). The results with the optimal frequency band and time period are shown in Figure 6(c)-(d). It is observed that in the object-directed scenario, the features of the left and right hand MI could be better discriminated than those in the non-objectdirected scenario, which is evident from the observation that the distribution of two group samples in Figure 6(b) is scattered more widely than that shown in Figure 6(a). A similar observation is also made from comparing Figure 6(c) and (d). In addition, when comparing the results in Figure 6(a)-(b) and Figure 6(c)-(d), we further notice that by applying the optimal frequency band and time period in both scenarios, the scatter of samples becomes more obvious so that they are clearly divided into left and right hand MI classes.

\subsection{Classification performance}

The cross-validation and testing results for different subjects and scenarios without optimization are shown in Table 4, and the corresponding results with optimization are shown in Table 5. After applying the optimal frequency band and time period, the average performances in cross-validation over the subjects increased from $76.11 \% \pm 5.30 \%$ to $83.01 \% \pm 4.57 \%$ (the maximum is $93.91 \%$ ) in object-directed $\mathrm{MI}$ and from $73.72 \% \pm 5.66 \%$ to $78.03 \% \pm 4.97 \%$ in non-object-directed MI. The average performances in the testing with optimization were $76.87 \% \pm 5.92 \%$ and $69.66 \% \pm 6.45 \%$, respectively. Comparing the results in Table 4 and Table 5, we can conclude that the classification performance would improve with the optimization of the frequency band and time period. The results further demonstrate that the classification performance is better in the 


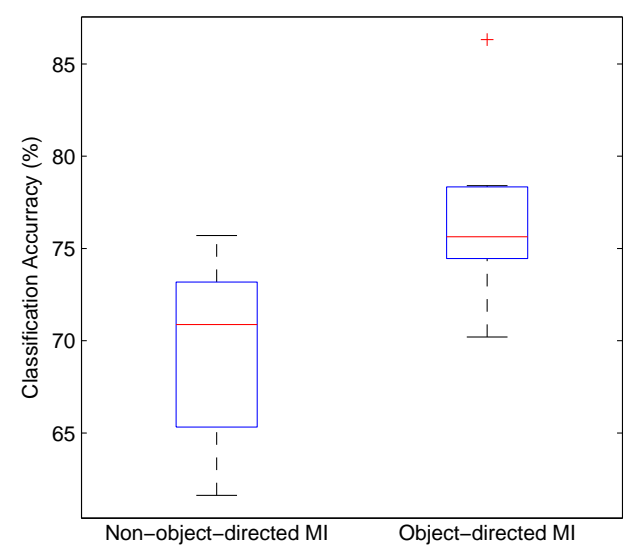

Figure 7: A comparison of the classification accuracy distributions in the nonobject-directed and object-directed MI.

object-directed scenario. In the non-object-directed scenario, the classification accuracy was in the range of $60 \%$ and $75 \%$ for the majority of the subjects. The accuracy was higher than $75 \%$ for one of the subjects. On the other hand, as shown in Table 5, in the object-directed scenario, the classification accuracy was in the range of $75 \%$ to $80 \%$ for most of the subjects, while the accuracy could even reach $85 \%$ or higher. It is found that for subject 5 , the classification performance was the worst among the subjects, which could be due to fatigue caused by the repeated experimental processes.

We further evaluate the classification performance in the object-directed and the non-object-directed scenarios by performing the t-test. A statistically significant difference is found between the two scenarios ( $p$-value is 0.0207), indicating that the object-directed scenario can improve the classification accuracy. Figure 7 shows a comparison of the classification accuracy distribution of the two groups, using a box plot. We present the classification accuracy distribution using its quartile, minimum, maximum, and, median from all subjects.

\section{Discussions}

This study was conducted expressly to investigate the effec$\mathrm{t}$ on the performance of MI-based BCIs using virtual objectdirected movements as visual guidance. We hypothesized that the modulation of sensorimotor EEG rhythms in the objectdirected scenario can improve classification accuracy and is thus beneficial for the development of MI-based BCIs and the training protocols. Most previous studies focused on the development of signal processing algorithms to improve the performance of BCI in distinguishing human intentions, e.g. messages and commands. However, the success of BCIs also depends on the effectiveness of the training approaches. For MIbased BCIs, it is essential to design effective training scenarios to enhance ERD features for to accurately identify different MI tasks. In this regard, we designed virtual scenarios of hand movements in an attempt to enhance the discrimination of MI tasks. The results show that our approach has improved user training and thus the performance of MI-based BCIs. We have demonstrated that under the object-directed scenario, contralateral dominant ERD patterns produced by MI are more readily recognizable for the discrimination of left and right hand MI tasks, when compared with that under the non-object-directed scenario.

The average classification accuracy increased by about $7 \%$ when the subjects performed MI with object-directed movements provided as visual guidance. The result of the t-test indicates that the improvement in classification performance under the object-directed scenario was statistically significant $(p=0.0207)$. These results are also better than the ones reported in a recent study (Ono et al., 2013), which suggests that the proposed approach could be used to improve MI-based BCI training.

As the main objective of this study is to investigate the effect of using object-oriented movements in a virtual environmen$t$ as visual guidance on the modulation of sensorimotor EEG rhythms generated by hand MI, we employed a standard LDA to classify left and right hand MI due to its generalization and efficacy in practical applications. In recent years, researchers have proposed a few more advanced methods based on the standard LDA in order to achieve better classification performance. To make a more comprehensive comparison, we implemented three advanced versions of LDA, including sparse LDA (SLDA) (Shin et al., 2012), shrinkage LDA (SKLDA) (Vidaurre et al., 2009; Blankertz et al., 2011), and Bayesian LDA (BLDA) (Hoffmann et al., 2008), to classify left and right hand $\mathrm{MI}$ in our experiments. For the non-object-directed scenario, the classification accuracies are $70.54 \pm 4.71 \%, 71.90 \pm 4.45 \%$ and $72.20 \pm 4.21 \%$ for SLDA, SKLDA, and BLDA respectively, while for the object-directed scenario, the classification accuracies are $77.22 \pm 7.47 \%, 77.96 \pm 7.18 \%$ and $78.13 \pm 7.21 \%$ for SLDA, SKLDA, and BLDA respectively. Compared with the results of the standard LDA $(69.66 \pm 6.45 \%$ and $76.87 \pm 5.92 \%$ for the non-object-directed scenario and the object-directed scenario, respectively), these advanced methods achieved somewhat better classification accuracy. However, regardless of what classifier we employed, the classification accuracy of the object-directed scenario is always obviously better than that of the non-object-directed scenario, further demonstrating that well-designed visual guidance can help improve the performance of MI-based BCI applications. In principle, these advanced methods can achieve much better performance than the standard LDA when the feature vector has a high dimension and the training samples are quite limited, which was not the case in our experiments. Therefore, the standard LDA is sufficient in our application. Moreover, recently, some new approaches have been put forward to improve the classification performance under the condition of insufficient training samples, such as spatial-temporal discriminate analysis (STDA) (Zhang et al., 2013b) and the aggregation of sparse linear discriminant analysis (ASLDA) (Zhang et al., 2014b). In the future, we shall further investigate if these algorithms can further improve the performance of MI-based BCI applications under virtual reality based visual guidance. 
Apart from studying the experimental training settings for MI-based BCI systems, we have also developed an algorithm to increase the accuracy of EEG pattern recognition. The algorith$\mathrm{m}$ deals with inter-subject variations in MI tasks by extracting subject-specific optimal time and frequency bands for enhancing the discrimination of ERD patterns. The results show that for both the object-directed and non-object-directed scenarios, most of the ERD patterns recorded during left and right hand MI remained around $10-12 \mathrm{~Hz}$ (in the upper $m u 2$ band). From the optimal time periods obtained with the proposed algorithm, as shown in Table 3, better classification results can generally be achieved by extracting the signals from the middle portion of the MI period. The feature vector distribution shown in Figure 4 further illustrates that the distinctions in ERD patterns can be enhanced by using the optimal time and frequency bands identified with our algorithm.

In our experimental paradigm, subjects were guided in the performance of MI tasks by providing them with continuous visual hand movements as displayed on the computer screen, and with auditory cues. The trigger signals of the paradigm defined and controlled the timing and duration of the MI period and the rest period. Indeed, a practical BCI system should be asynchronous and capable of detecting the user's intention at any time. Owing to the difficulty in carrying out real-time detections of the MI periods and rest periods, asynchronous BCI systems have not yet become widely available. Nevertheless, the continuous EEG signals obtained from our experiment could be employed by asynchronous BCI systems to detect the switching of MI states.

\section{Conclusion}

This study indicates that the use of object-directed movement as visual guidance is an effective approach for training in MI-based BCI systems. The technique of extracting subjectspecific time and frequency bands can be applied to enhance the discrimination accuracy of hand MI. The proposed methods and the findings are therefore helpful for the future development of BCI applications. In this study, the experiments were carried out on healthy subjects. A further study will be conducted to perform the same experimental paradigm on people with movement disorders. It has also been reported that, in addition to hand movements, distinguishable EEG patterns in the $m u$ rhythm can be obtained during MI tasks involving tongue, leg and feet movements (Pfurtscheller et al., 2006). Our experimental paradigm will be extended to study the effect of visual guidance on these MI tasks.

\section{References}

Arvaneh, M., Guan, C., Ang, K. K., \& Quek, C. (2013). Optimizing spatial filters by minimizing within-class dissimilarities in electroencephalogrambased brain-computer interface. Neural Networks and Learning Systems, IEEE Transactions on, 24, 610-619.

Babiloni, C., Babiloni, F., Carducci, F., Cincotti, F., Cocozza, G., Del Percio, C., Moretti, D. V., \& Rossini, P. M. (2002). Human cortical electroencephalography (eeg) rhythms during the observation of simple aimless movements: a high-resolution eeg study. Neuroimage, 17, 559-572.
Blankertz, B., Lemm, S., Treder, M., Haufe, S., \& Müller, K.-R. (2011). Singletrial analysis and classification of erp componentsła tutorial. NeuroImage, $56,814-825$.

Daly, J. J., \& Wolpaw, J. R. (2008). Brain-computer interfaces in neurological rehabilitation. The Lancet Neurology, 7, 1032-1043.

Duda, R. O., Hart, P. E., \& Stork, D. G. (2001). Pattern classification. 2nd. Edition. New York.

Ertelt, D., Small, S., Solodkin, A., Dettmers, C., McNamara, A., Binkofski, F., \& Buccino, G. (2007). Action observation has a positive impact on rehabilitation of motor deficits after stroke. Neuroimage, 36, T164-T173.

Higashi, H., \& Tanaka, T. (2013). Simultaneous design of fir filter banks and spatial patterns for eeg signal classification. Biomedical Engineering, IEEE Transactions on, 60, 1100-1110.

Hoffmann, U., Vesin, J.-M., Ebrahimi, T., \& Diserens, K. (2008). An efficient p300-based brain-computer interface for disabled subjects. Journal of Neuroscience methods, 167, 115-125.

Huang, N. E., Shen, Z., Long, S. R., Wu, M. C., Shih, H. H., Zheng, Q., Yen, N.-C., Tung, C. C., \& Liu, H. H. (1998). The empirical mode decomposition and the hilbert spectrum for nonlinear and non-stationary time series analysis. Proceedings of the Royal Society of London. Series A: Mathematical, Physical and Engineering Sciences, 454, 903-995.

Jackson, P. L., Lafleur, M. F., Malouin, F., Richards, C., \& Doyon, J. (2001). Potential role of mental practice using motor imagery in neurologic rehabilitation. Archives of physical medicine and rehabilitation, 82, 1133-1141.

Jasper, H. H. (1958). The ten twenty electrode system of the international federation. Electroencephalography and clinical neurophysiology, 10, 371375.

Leocani, L., Toro, C., Manganotti, P., Zhuang, P., \& Hallett, M. (1997). Eventrelated coherence and event-related desynchronization/synchronization in the $10 \mathrm{hz}$ and $20 \mathrm{hz}$ eeg during self-paced movements. Electroencephalography and Clinical Neurophysiology/Evoked Potentials Section, 104, 199-206.

McFarland, D. J., McCane, L. M., David, S. V., \& Wolpaw, J. R. (1997). Spatial filter selection for eeg-based communication. Electroencephalography and clinical Neurophysiology, 103, 386-394.

McFarland, D. J., Miner, L. A., Vaughan, T. M., \& Wolpaw, J. R. (2000). Mu and beta rhythm topographies during motor imagery and actual movements. Brain topography, 12, 177-186.

Muthukumaraswamy, S. D., Johnson, B. W., \& McNair, N. A. (2004). Mu rhythm modulation during observation of an object-directed grasp. Cognitive Brain Research, 19, 195-201.

Neuper, C., \& Pfurtscheller, G. (2001). Event-related dynamics of cortical rhythms: frequency-specific features and functional correlates. International journal of psychophysiology, 43, 41-58.

Neuper, C., Scherer, R., Wriessnegger, S., \& Pfurtscheller, G. (2009). Motor imagery and action observation: modulation of sensorimotor brain rhythms during mental control of a brain-computer interface. Clinical neurophysiology, 120, 239-247.

Oberman, L. M., McCleery, J. P., Ramachandran, V. S., \& Pineda, J. A. (2007). Eeg evidence for mirror neuron activity during the observation of human and robot actions: Toward an analysis of the human qualities of interactive robots. Neurocomputing, 70, 2194-2203.

Ono, T., Kimura, A., \& Ushiba, J. (2013). Daily training with realistic visual feedback improves reproducibility of event-related desynchronisation following hand motor imagery. Clinical Neurophysiology, .

Peter, B., Ashley, C., Yvonne, T., \& Hung, N. (2010). Discrimination of left and right leg motor imagery for brain-computer interfaces. Med Biol Eng Comput, 48, 343-350.

Pfurtscheller, G., Brunner, C., Schlögl, A., \& Lopes da Silva, F. (2006). Mu rhythm (de) synchronization and eeg single-trial classification of different motor imagery tasks. NeuroImage, 31, 153-159.

Pfurtscheller, G., \& Neuper, C. (1997). Motor imagery activates primary sensorimotor area in humans. Neuroscience letters, 239, 65-68.

Pfurtscheller, G., Neuper, C., Flotzinger, D., \& Pregenzer, M. (1997). Eegbased discrimination between imagination of right and left hand movement. Electroencephalography and Clinical Neurophysiology, 103, 642-651.

Pfurtscheller, G., Neuper, C., \& Krausz, G. (2000). Functional dissociation of lower and upper frequency mu rhythms in relation to voluntary limb movement. Clinical neurophysiology, 111, 1873-1879.

Pfurtscheller, G., Neuper, C., Ramoser, H., \& Müller-Gerking, J. (1999). Visually guided motor imagery activates sensorimotor areas in humans. Neuroscience letters, 269, 153-156. 
Pfurtscheller, G., Neuper, C., Schlogl, A., \& Lugger, K. (1998). Separability of eeg signals recorded during right and left motor imagery using adaptive autoregressive parameters. Rehabilitation Engineering, IEEE Transactions on, 6, 316-325.

Pfurtscheller, G., Scherer, R., Leeb, R., Keinrath, C., Neuper, C., Lee, F., \& Bischof, H. (2007). Viewing moving objects in virtual reality can change the dynamics of sensorimotor eeg rhythms. Presence: Teleoperators and Virtual Environments, 16, 111-118.

Pfurtscheller, G., \& Lopes da Silva, F. (1999). Event-related eeg/meg synchronization and desynchronization: basic principles. Clinical neurophysiology, 110, 1842-1857.

Prasad, G., Herman, P., Coyle, D., McDonough, S., \& Crosbie, J. (2010). Applying a brain-computer interface to support motor imagery practice in people with stroke for upper limb recovery: a feasibility study. J Neuroeng Rehabil, 7, 60.

Rizzolatti, G., Fadiga, L., Gallese, V., \& Fogassi, L. (1996). Premotor cortex and the recognition of motor actions. Cognitive brain research, 3, 131-141.

Shin, Y., Lee, S., Lee, J., \& Lee, H.-N. (2012). Sparse representation-based classification scheme for motor imagery-based brain-computer interface systems. Journal of neural engineering, 9, 056002.

Vidaurre, C., Krämer, N., Blankertz, B., \& Schlögl, A. (2009). Time domain parameters as a feature for eeg-based brain-computer interfaces. Neural Networks, 22, 1313-1319.

Wolpaw, J. R., Birbaumer, N., McFarland, D. J., Pfurtscheller, G., \& Vaughan, T. M. (2002). Brain-computer interfaces for communication and control. Clinical neurophysiology, 113, 767-791.

Zhang, Y., Zhou, G., Jin, J., Wang, M., Wang, X., \& Cichocki, A. (2013a). L1-regularized multiway canonical correlation analysis for ssvep-based bci. Neural Systems and Rehabilitation Engineering, IEEE Transactions on, 21, 887-896.

Zhang, Y., Zhou, G., Jin, J., Wang, X., \& Cichocki, A. (2014a). Frequency recognition in ssvep-based bci using multiset canonical correlation analysis. International journal of neural systems, 24, 1450013.

Zhang, Y., Zhou, G., Jin, J., Wang, X., \& Cichocki, A. (2015). Optimizing spatial patterns with sparse filter bands for motor-imagery based braincomputer interface. Journal of neuroscience methods, 255, 85-91.

Zhang, Y., Zhou, G., Jin, J., Zhao, Q., Wang, X., \& Cichocki, A. (2014b). Aggregation of sparse linear discriminant analyses for event-related potential classification in brain-computer interface. International journal of neural systems, 24, 1450003.

Zhang, Y., Zhou, G., Zhao, Q., Jin, J., Wang, X., \& Cichocki, A. (2013b). Spatial-temporal discriminant analysis for erp-based brain-computer interface. Neural Systems and Rehabilitation Engineering, IEEE Transactions on, 21, 233-243. 